\title{
Reflections on thermoelectrics
}

\author{
On the twentieth anniversary of two influential papers we consider past developments and future \\ opportunities for thermoelectric materials.
}

In May and June of 1993, Lyndon Hicks and Mildred Dresselhaus published two theoretical papers that showed reducing the dimensionality of thermoelectric materials could dramatically improve their performance $^{1,2}$. In this issue, we publish a Commentary by Joseph P. Heremans, Mildred S. Dresselhaus, Lon E. Bell and Donald T. Morelli on the history and legacy of this work ${ }^{3}$. The authors explain that the two papers emerged from a need to provide a new perspective on a field, which, at the time, seemed to have stalled.

Thermoelectrics can, in principle, transform wasted heat into electricity. Conversely, an electrical voltage applied to a thermoelectric array can be used to produce local heating or cooling. It is easy to dream about power generators using wasted heat, or refrigerators based on small thermoelectric components. However, to be widely used thermoelectrics need to be efficient. The efficiency is measured by the figure of merit, $Z T$, which is proportional to the electrical conductivity and inversely proportional to the thermal conductivity. Because the two quantities are interdependent, it is a challenge to achieve values of $Z T$ necessary for large-scale applications.

Before 1993, research in thermoelectrics was primarily aimed at finding new materials that would exhibit the highest $Z T$, and the best results were obtained with doped Bi-Te compounds. The work by Hicks and Dresselhaus suggested that in two- and one-dimensional semiconductors, $Z T$ could be enhanced by an order of magnitude due to modifications of the electronic density of states and the enhanced scattering of phonons.

The two papers undoubtedly re-invigorated the field, and produced several improvements. On reflection, however, progress in terms of improvements of $Z T$ has been slow. Some of the higher values reported have not been easy to reproduce. Furthermore, applications have remained limited to specific fields. One example is power generation in space vehicles, such as the power generator in the Mars Curiosity rover. Another is the Climate Control Seat for motor vehicles made by the company Gentherm.

The success of research in thermoelectrics should, however, not be measured exclusively

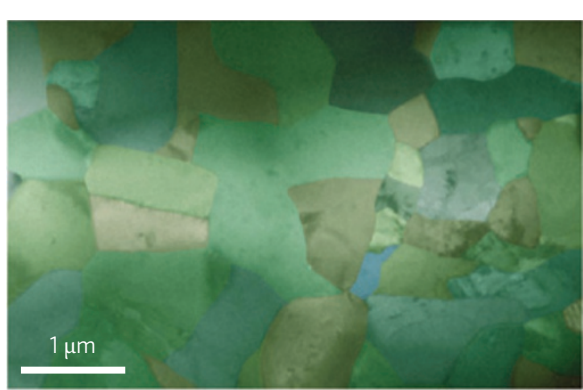

be possible, however, is the development of functionalities and applications that are unique to these materials. As in the case of the Curiosity rover, it is possible to imagine other self-powered controls or sensors. Alternatively, as in the case of the Climate Control Seat, it is possible to picture other compact devices for on-demand thermal management in home and commercial furnishings.

Twenty years ago, the work of Hicks

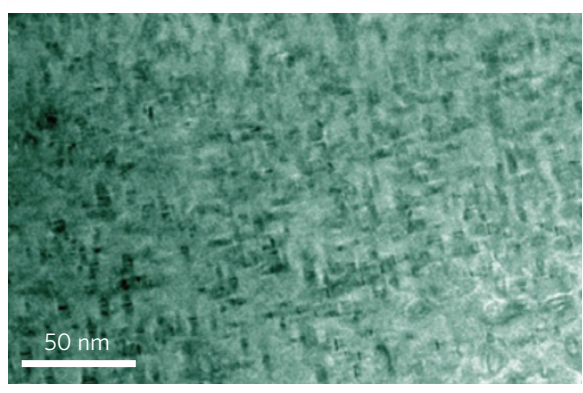
and Dresselhaus provided a new approach to thermoelectrics by suggesting that the morphology of a thermoelectric system can be used to both improve the electronic transport and increase the phonon scattering. In the past few years, most research efforts have been aimed at reducing the thermal conductivity without affecting the electron transport. Although this will, in the future, provide additional incremental advances, there is
Nanostructures at different length scales in $\mathrm{PbTe}$ provide efficient phonon scattering. Image reproduced from ref. 4, (c) 2012 NPG.

in terms of simplistic goals like a rapid development of high ZT materials or the creation of power generators that can substitute existing ones. The search for high ZT materials has also led to significant advances in fundamental science. In particular, there have been improvements in the control of the material structure as well as in the understanding of electron and phonon transport properties.

Such improvements have fed back into the development of high ZT materials. For example, efficient scattering of phonons in PbTe has been obtained by using nanoscale structuring at different length scales ${ }^{4}$. Another important advance has been the development of the phonon-glass/electron-crystal concept by Glen Slack ${ }^{5}$, which was then used to describe the low thermal conductivity in several materials including skutterudites and clathrates. Furthermore, excellent results have been obtained in $\mathrm{Pb}-\mathrm{Te}-\mathrm{Se}$ materials by increasing the electrical conductivity through distortion of the electronic bands $s^{6,7}$.

From an applications point of view, thermoelectrics will not, for example, solve the energy crisis anytime soon. What might now a need to also enhance the electrical conductivity. The work on $\mathrm{Pb}-\mathrm{Te}-\mathrm{Se}$ materials is a good example of this approach $^{6,7}$. Another possibility is to explore the potential advantages provided by metallic states at the surfaces of topological insulators ${ }^{8,9}$.

Finally, one intriguing future direction is the possibility of generating electric voltage through using the effect a thermal gradient has on the electron spin. This field, which is known as spin caloritronics ${ }^{10}$, has already provided a variety of fundamental insights, and in the future, collaborations between experts in spintronics and thermoelectrics could lead to the development of new applications.

References

1. Hicks, L. D. \& Dresselhaus, M. S. Phys. Rev. B 47, 12727-12731 (1993)

2. Hicks, L. D. \& Dresselhaus, M. Phys. Rev. B 47, 16631-16634 (1993).

3. Heremans, J. P., Dresselhaus, M. S., Bell, L. E. \& Morelli, D. T. Nature Nanotech. 8, 471-473 (2013).

4. Biswas, K. et al. Nature 489, 414-418 (2012).

5. Slack, G. A. in CRC Handbook of Thermoelectrics (ed. Rowe, D. M.) 407-440 (CRC, 1995).

6. Heremans, J. P. et al. Science 321, 554-557 (2008).

7. Pei, Y., Shi, X., Lalonde, A., Wang, H., Chen, L. \& Snyder, G. J. Nature 473, 66-69 (2011).

8. Ghaemi, P., Mong, R. S. K. \& Moore, J. E. Phys. Rev. Lett. 105, $166603(2010)$

9. Tretiakov, O. A., Abanov, Ar., Murakami, S. \& Sinova, J. Appl. Phys. Lett. 97, 073108 (2010).

10. Bauer, G. E. W., Saitoh, E. \& van Wees, B. J. Nature Mater. 11,391-399 (2012). 